\title{
Detecting change in the urban road environment along a route based on traffic sign and crossroad data
}

\author{
Zoltán Fazekas, Gábor Balázs, László Gerencsér and Péter Gáspár \\ Institute for Computer Science and Control (MTA SZTAKI), \\ Budapest, H-1111, Kende u. 13-17, Hungary \\ \{zoltan.fazekas, gabor.balazs, laszlo.gerencser, peter.gaspar\} \\ @sztaki.mta.hu
}

\begin{abstract}
Occurrences of traffic signs that belong to certain sign categories and occurrences of crossroads of various topologies are utilized in detecting change in the urban road environment that moves past an ego-car. Three urban environment types, namely downtown, residential and industrial/commercial areas, are considered in the study and changes between these are to be detected. In the preparatory phase, the ego-car is used for traffic sign and crossroads data collection. In the application phase, the ego-car hosts an advanced driver assistance system (ADAS) that captures and analyzes images of the road environment and computes the required input data to the proposed road environment detection (RoED) subsystem. A statistical inference method relying on the minimum description length (MDL) principle was applied to the change detection problem at hand. The above occurrences along a route are seen as a realization of an inhomogeneous marked Poisson process. Page-Hinkley change detectors tuned to empirical data were set to work to detect change in the urban road environment. The process and the quality of the change detection are demonstrated via examples from three urban settlements in Hungary.
\end{abstract}

Keywords: Urban environment types; Detection of road environment; Marked Poisson point process; Statistical change detection; The minimum description length principle; Page-Hinkley detectors; Advanced driver assistance systems; Traffic sign recognition systems.

\section{Introduction}

Drivers are assisted in many ways and via different media - ranging from the paperbased maps, through road and traffic signs, electronic information boards and emergency radio broadcasts to pictorial, navigational, and textual information services - in perceiving and understanding driving conditions and road environment ahead and around them. By using these facilities and media, they get access to a wide variety of road, weather, traffic and accident information, though the relevance, timeliness and accuracy of the provided information varies across the palette. For an analysis of the utility of the various facilities and media available for the purpose, particularly of the more recent (i.e., electronic) services, relevance, see [1] and [2]. 
The drivers driving smart cars also benefit from the various intelligent functions, such as the traffic sign recognition (TSR), the lane keeping assistance and the road marking recognition functions, offered by the respective and other high-end subsystems installed within and on-board their cars. Such subsystems together constitute the advanced driving assistance systems (ADAS). The ADAS as complete systems - and also their specific subsystems - are designed to increase driver and passenger safety, and in many cases also the driver and passenger comfort. An edifying presentation of an $A D A S$ system architecture that provides a comprehensive set of ADAS functions is given in [3].

Herein, traffic sign data gathered, categorized and logged by human data entry assistants, or by on-board TSR systems, or, alternatively, extracted from road-related geographical information systems (GIS), crossroad data gathered, categorized and recorded by data entry assistants, or detected, combined, analyzed, categorized and recorded by various ADAS subsystems ${ }^{1}$, or, alternatively, extracted from some road-related GIS are utilized in detecting change in the type of road environment along a route within an urban settlement.

It should be noted that in all the concrete cases presented herein, both the traffic sign and crossroad detections - together with the categorization and data logging tasks were carried out by data entry assistants. Later, however, and considering the pace of the development of the ADAS capabilities probably in the near future, these activities could be left to the respective ADAS subsystems. Furthermore, these subsystems might also take advantage of the information that can be extracted from road-related GIS, e.g., the crossroad data could be extracted from the roads layer of the relevant Open Street Map (OSM) [5]. To illustrate this point, the mentioned layer - together with two other ones - are displayed in the map² of Vác (Fig. 1).

\subsection{The road environment detection and other ADAS capabilities}

Let us now analyze the tasks of identifying the current urban road environment type and of detecting the transitions between these from an ADAS capabilities viewpoint. Firstly, as it was mentioned above, certain object recognition and data gathering, logging and analysis tasks could be left to ADAS subsystems (e.g., to the TSR subsystems).

Secondly, the type of the current urban road environment - and the transitions between different urban road environments - constitute relevant information for the drivers, as different road environments pose different safety risks, and consequently the drivers should look out for different things, events and situations in these environments.

1 Including also the TSR subsystem, which per se could be used in gathering, categorizing and logging traffic sign data, but perhaps its connection to activities in respect of crossroad data needs to be construed. The connection between the TSR system and the crossroad data is embodied in certain traffic sign types, e.g., $\rightarrow, A$ and $\boldsymbol{A}$ traffic signs. The automatic recognition of lane information traffic signs for ADAS purposes was addressed in [4].

2 The map was created with the QGIS (version 2.8) software, which is a free and open source GIS tool. 
For example, one should drive more cautiously in a densely populated busy downtown area, than, say, in a calm residential area with virtually no traffic. Also, drivers should be more prepared to see and patiently tolerate a flow of heavy vehicles in an industrial/commercial area than in a downtown environment.

For the above reasons many a driver would welcome some computerized assistance - expediently in the form of a dedicated ADAS function/subsystem - in respect of the aforementioned tasks. In response to this demand a road environment detection (RoED) subsystem was proposed in [6]. Its actual implementation targets rural and intercity road environments. It relies on advanced perception methodology and up-to-date vehicle automation techniques, as well as recent inter-vehicular and vehicle-to-infrastructure communication framework to achieve real-time detection and classification of obstacles and to identify other potential risks also in real-time.

A different RoED approach that uses traffic sign data as input was proposed in [7] and the associated method is now extended and developed herein. The proposed method relies on the availability of an on-board TSR subsystem and was developed for cars equipped with such a subsystem. Such a TSR-based RoED subsystem could be seen as a low-cost surrogate for the comprehensive computer vision-based road environment understanding and recognition systems, such as the one proposed in [6].

Thirdly, a further interesting ADAS aspect that should be considered here is the feedback from the RoED subsystem back to the invoked ADAS subsystems. For instance, the RoED subsystem could facilitate the computer vision and the image understanding computations (e.g., in the TSR subsystem) by providing geometrical constraints and regions-of-interests for them; thereby making the various recognitions and visual measurements more robust and reliable.

\section{Related work}

The research presented herein fits in the series of papers [7], [8] and [9] on statistical change detection problems pertaining various road environments and using traffic signrelated data as input. In [8], a statistical inference method for detecting a transition between two different topographical road environments when driving from one environment to the other was presented. Logs of traffic sign occurrences recording the traffic sign locations and types were analyzed. The somewhat impractical task targeted in the paper (i.e., finding the piedmont via looking at traffic signs along a route) was seen as a test case for the applied minimum description length (MDL) methodology. In [7], the change of the urban scenery - often experienced when driving from the periphery of a town to its centre, or the other way round - is traced in traffic sign logs. The task there was to identify the change-point between different urban road environments. The statistical approach infers the type of the urban environment the car is being driven in and detects the transitions between these road environments from the traffic sign occurrences and from the corresponding location and categorical data along a route. The traffic signs considered belong to a pre-selected class. The RoED method relies on empirical data and the MDL principle is applied to solve the problem at hand. The method makes use of several Page-Hinkley change detectors (PHCD's). Some improvement in the detection performance could be achieved by considering an augmented set of inputs. 
To check the validity of this expectation, the crossroad types and locations were included in the input data. The resulting urban RoED method is presented herein.

\section{Mathematical background}

Marked point process. Marked point process is a convenient model for describing categorized data, e.g., the traffic sign and crossroad data used and analysed herein. A point process is customarily given by an increasing sequence of time points ${ }^{3}$, say $T_{n}$. The points of a point process may be labelled with marks. A marked point process then can be formalized as a pair $\left(T_{n}, \rho_{n}\right)$, where $\rho_{n}$ is the mark. In many real-world cases that can be modelled with marked point processes, the marked Poisson point processes (MPPP) turned out to be convenient and flexible stochastic models. Although the Poisson process, and also the MPPP, are continuous-time ${ }^{4}$ models, their discrete-time approximations ${ }^{5}$ are commonly used, as it is also done herein.

Change detection. The problem of detecting abrupt changes in the dynamics of stochastic signals is widely discussed in the literature. Also, many interesting applications were analyzed in this respect. Change detection within independent and identically distributed (iid) random data was targeted first some fifty-sixty years ago. This effort led to the Page-Hinkley change detector ${ }^{6}$ (PHCD), see [10], [11] and [12], which has been used widely in the field ever since. The most important performance criteria concerning change detectors are the average run length between false alarms and the expected delay in detection.

Minimum description length principle. An interesting approach to change detection in the dynamics of stochastic signals is based on the minimum description length (MDL) principle $^{7}$. The approach was proposed in [16] and later elaborated in [17].

The approach was used with success in tasks ranging from model selection through feature extraction to summarizing tasks, see e.g., [18]. Its essence is to choose between stochastic models - for the purpose of describing data - according to the minimum codelength that can be achieved by encoding the data (relying on the above models). The advantage of the MDL methodology is its great flexibility (e.g., the PHCD can be seen as a procedure relying on MDL approach).

\footnotetext{
3 Spatial points rather than temporal points can be used. Herein: the path-length covered by egocar will be used as a sequencing parameter.

4 Herein: continuous space model.

5 Herein: discrete-space approximation.

6 The PHCD was later adopted and analysed also for dependent data [13].

7 The MDL principle has its theoretical foundations in information theory. The principle was proposed in [14] and later developed further in [15].
} 
Page-Hinkley change detector. Assume that a sequence of observations $\xi_{1}, \ldots, \xi_{N}$ is given. Furthermore, assume that it is composed of two parts: the first part being an iid sequence of random variables taking discrete values according to the probability law $p\left(\xi_{n}, \theta_{1}\right)$, while the second part being generated according to another probability law $p\left(\xi_{n}, \theta_{2}\right)$ in a similar manner. The problem is to estimate the time, or the location, of the change between the two probability laws from observed data in a real time manner.

An MDL approach to solve this problem would be to choose an arbitrary time - or location $-\tau$ and assuming that this is when, or where, the transition between the probability laws takes place, encode the observed data optimally according to the hypothesized data generating mechanism. According to standard results of information theory, the overall optimal code-length $L_{N}(\tau)$ of the observed data - in an asymptotic sense and allowing block coding - is as follows:

$$
L_{N}(\tau)=\sum_{n=1}^{\tau-1}-\log p\left(\xi_{n}, \theta_{1}\right)+\sum_{n=\tau}^{N}-\log p\left(\xi_{n}, \theta_{2}\right) .
$$

According to the MDL principle, the estimator of the change point is obtained by minimizing $L_{N}(\tau)$ in $\tau$. In order to explore the shape of $L_{N}$ as $\tau$ varies, one could compare its successive values, e.g., $L_{N}(\tau)$ and $L_{N}(\tau+1)$, and could use them to define the score $\Delta L_{N}(\tau)$ as

$$
\Delta L_{N}(\tau)=L_{N}(\tau+1)-L_{N}(\tau) .
$$

A heuristic procedure for minimizing $L_{N}$ in real-time is obtained by identifying the time-point, or space-point, after which $L_{N}$ has a clear upward trend. Then the following signal needs to be monitored:

$$
g_{n}=S_{n}-\min _{m \leq n} S_{n}, \text { where } S_{n}=\sum_{k=1}^{n} \Delta L_{N}(k) .
$$

It is then expected that $g_{n}$ is typically 0 for $n$ 's preceding the true change point, while $g_{n}$ is typically increasing after that. Choosing a positive threshold for $g_{n}$ to surpass, the corresponding $n$ will mark the estimator of the change point. This heuristic procedure can be interpreted as and implemented using a PHCD that detects change in the parameter of the probability law according to which the observed data is generated.

\section{Collection of traffic sign and crossroad data}

Car-based data collection trips were made in respect of traffic signs, crossroads and urban environment types in three urban areas in Hungary. The areas involved were Csepel (one of the 23 districts of Budapest), Százhalombatta (a town on the west bank of the river Danube) and Vác (a historic town on the east bank of the River Danube). The trajectory of the data collection trip made to Vác is shown in Fig. 1, while photos of typical scenes from the mentioned settlements are presented in Fig. 2. Three different urban road environment types, namely downtown (Dt), residential (Res) and industrial/ 
commercial (Ind) areas ${ }^{8}$ were considered in the study, and changes between these were to be detected. The fact that certain types of traffic signs and crossroads tend to appear more/less frequently in the Dt areas, or in the Res areas, or in the Ind areas than they do in the other two, makes the statistical inference and the RoED possible. For the data collection trips a tablet-based Android application was developed to facilitate manual data entry in the moving car in respect of traffic signs, crossroads and urban environments encountered, and for geo-tagging the route in an on-going manner. Data logging was the task of a data entry assistant, who logged the data while the driver drove the car along a route.

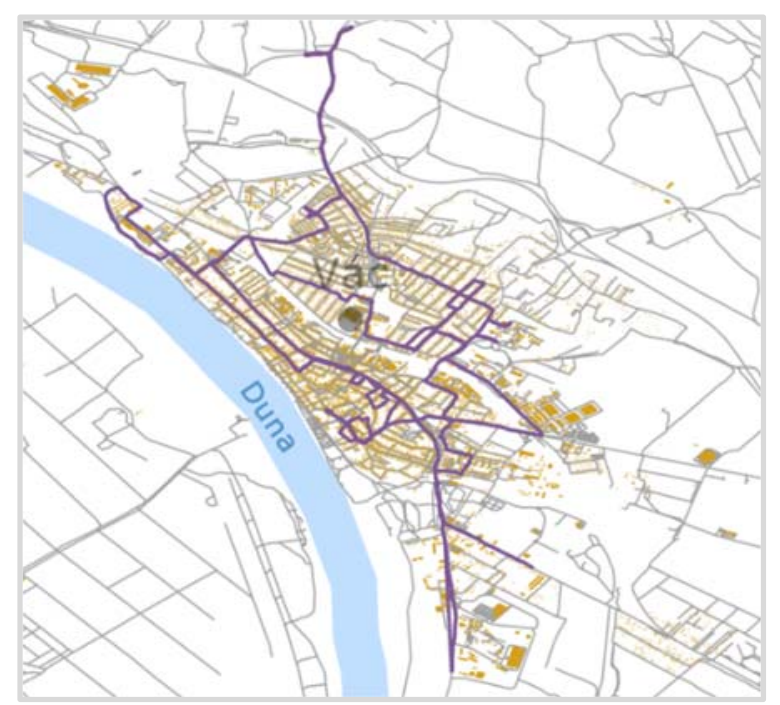

Fig. 1. The trajectory (purple polyline) of the data collection car trip made in Vác. The roads (grey lines) and the buildings (ochre shapes) layers and the River Danube, or Duna (light blue band) from the waterways layer of the OSM of Hungary are shown.

The empirical probabilities of encountering crossroads and traffic signs of various categories - over a path-length of $50 \mathrm{~m}$ - in different urban road environments are shown in the Figs. 3a and 3b, respectively. The crossroad types considered are as follows: T-shaped, X-shaped, complex crossroads and roundabouts - all these without traffic lights - and any crossroads with traffic lights installed. In the figure, these types are indicated with pictograms.

8 Downtown (Dt) areas feature one-, or multi-storey buildings built next to, or close to each other. Industrial/commercial (Ind) areas can be described in terms of factory buildings, workshops, stores - all of these with spacious yards - as well as supermarkets and bigger shops with parking lots. The residential (Res) areas are characterized by somewhat larger green spaces and by one- and two-storey buildings with somewhat more space between them than between those in the downtown areas. Note that there can be considerable variations in the descriptions among countries and regions. 

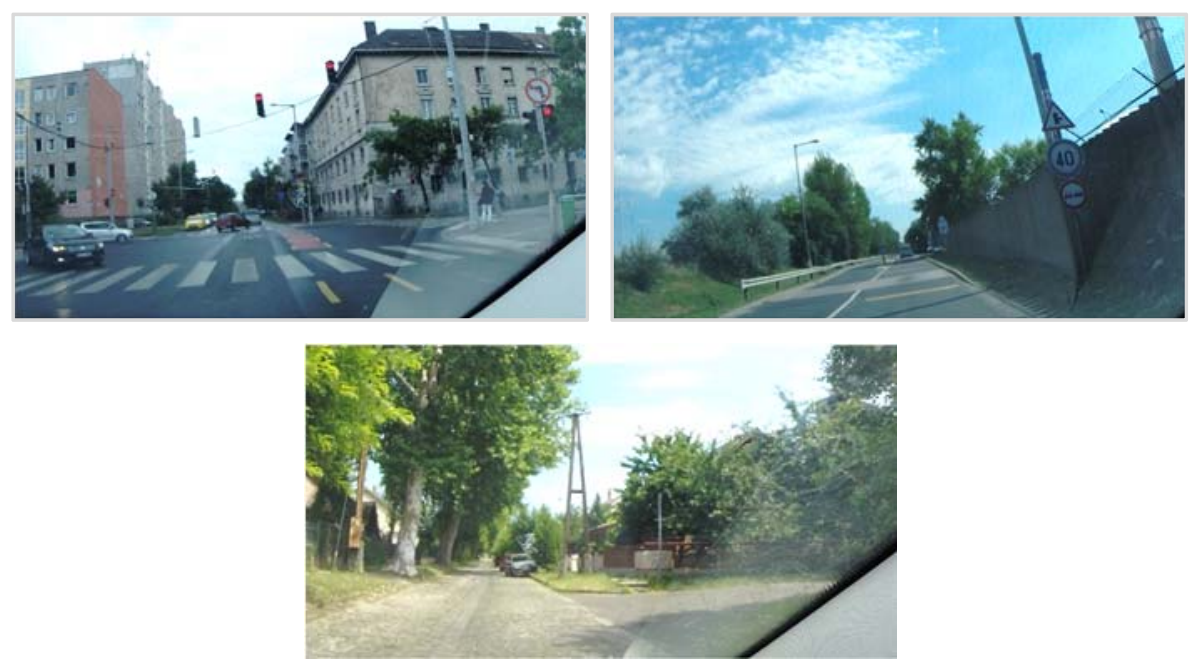

Fig. 2. A crossroad - with traffic lights - in Csepel's Dt (top left). A street in an Ind area in Százhalombatta (top right) and one in a Res area in Vác (bottom).
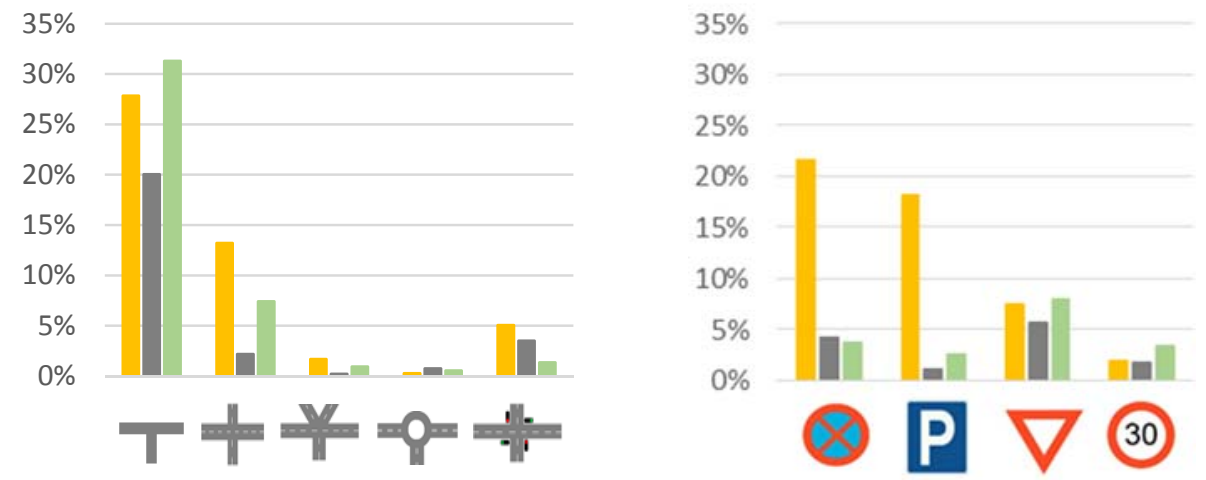

Fig. 3. Probabilities of encountering crossroads (left) and traffic signs (right) of the indicated types, respectively, over a path-length of $50 \mathrm{~m}$ in Dt (yellow), Ind (grey) and Res areas (green) based on the aggregated data.

\section{The proposed method for detecting change in urban environment}

A discrete time/space approximation of a MPPP was developed in [8]. It was obtained by assuming an inhomogeneous iid sequence of random variables with binomial distribution, taking values 1 and 0 , with probabilities $\theta_{i}$ and $1-\theta_{i}$, respectively.

In Eq. 4 , index $i$ refers to the sequential number associated with an urban road environment; say, Dt: 1 , Ind: 2 , and Res: 3 . In the equation, index $f$ refers to the road features 
considered, i.e., the traffic signs (ts) and the crossroads (cr); e.g., $\theta_{2}^{t s}$ is the probability that any kind of traffic sign occurs in an observation (i.e., over a route-segment of $50 \mathrm{~m}$ ) within an Ind area. An event occurs, if $\xi_{j}^{f}=1$, where $j$ identifies the observation; e.g., $\xi_{j}^{c r}$ signifies that a crossroad was detected in the $j$-th route-segment. The indicator functions $\zeta_{j, k}^{f}$ indicates the presence of a certain mark (i.e., type of the traffic sign or of the crossroad) in an observation; e.g., $\zeta_{j, 2}^{c r}$ signifies whether an X-shaped crossroad was detected in the $j$-th route-segment, or not. If one can assume that the stochastic processes describing the occurrences of traffic signs and crossroads are independent of each other, then the score obtained via optimally encoding the $j$-th observation using the two probability laws ${ }^{9}$ is as follows:

$\Delta L(j)=\sum_{f \in\{t s, c r\}}\left(-\xi_{j}^{f} \cdot \log \frac{\theta_{1}^{f}}{\theta_{2}^{f}}-\left(1-\xi_{j}^{f}\right) \cdot \log \frac{1-\theta_{1}^{f}}{1-\theta_{2}^{f}}-\sum_{k=1}^{m} \xi_{j}^{f} \cdot \zeta_{j, k}^{f} \cdot \log \frac{p_{1, k}^{f}}{p_{2, k}^{f}}\right)$.

This is simply the sum of the score derived for the traffic signs in [8] and the similar score for crossroads. Relying on the above formula and also on the corresponding formulae referred to in the footnote, one can compute based on Eq. 3 the signals to be monitored by the respective PHCD's.

\subsection{Examples for PHCD-based environmental change detections}

The outputs of PHCD's - used for detecting change in the urban road environment based on the collected data - are shown in Figs. 4 - 5 as concrete examples. These output signals were produced by the PHCD's were tuned to the empirical probabilities shown in Fig. 3.

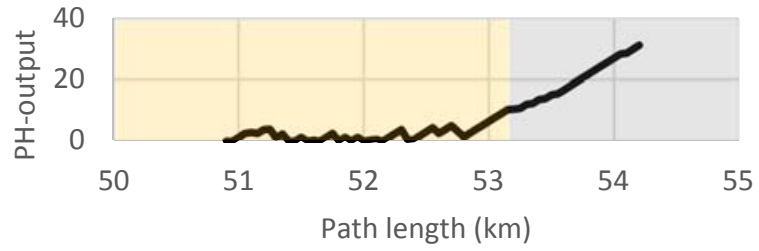

Fig. 4. An urban road environment change - encountered while driving from a Dt area in Csepel to an Ind area (Dt $\rightarrow$ Ind) - detected with a PHCD tuned to the overall empirical traffic sign and crossroad data. The yellow and the grey areas above correspond to the logged (i.e., true) Dt and Ind urban road environments.

\footnotetext{
${ }^{9}$ Note that Eq. 4 gives the score when comparing the optimal code-lengths corresponding to the $\mathrm{Dt}(1)$ and the Ind (2) areas, respectively. This is indicated, for instance, by the use of $\theta_{1}^{f}$ and $\theta_{2}^{f}$ in the formula. Eq. 4 corresponds to the case when one drives from a Dt area into an Ind area. Similar formulas can be derived for other ordered pairs of road environments.
} 


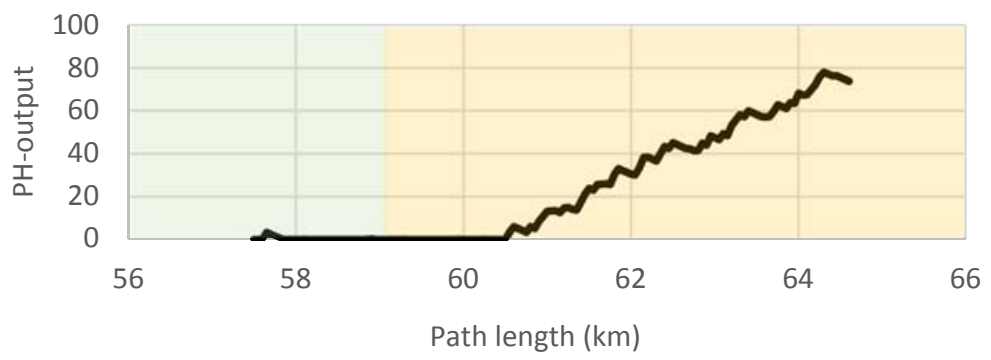

Fig. 5. An urban road environment change - encountered while driving from a Res area in Vác to a Dt area (Res $\rightarrow D t)$ - detected with a PHCD tuned to the overall empirical traffic sign and crossroad data. The green and the yellow areas above correspond to the logged (i.e., true) Res and Dt urban road environments.

Having been calculated from the mentioned empirical probabilities $\theta_{1}^{t s}=49 \%$, $\theta_{2}^{t s}=13 \%$ and $\theta_{3}^{t s}=18 \%$, as well as $\theta_{1}^{c r}=48 \%, \theta_{2}^{c r}=27 \%$ and $\theta_{3}^{c r}=42 \%$ were used in Eq. 4. Using these empirical probabilities and those displayed in Fig 3, the conditional probabilities $p_{1, k}^{f}$ can also be calculated. An environmental transition encountered when driving from a Dt area in Csepel to an Ind area is presented in Fig. 4, while one from a Res area in Vác to a Dt area is shown in Fig. 5 as examples.

\section{Conclusions}

When driving from the periphery of a town to its centre, or in the opposite direction for that matter, the urban scenery may change a lot. This changing scenery and more importantly the latent socio-economical, architectural, as well as transport- and trafficrelated changes behind the visible traits pose highly different safety risks to the drivers and it is important that they are aware of these risks. To assist drivers in perceiving and understanding the road environment, a TSR-based surrogate RoED ADAS-function was proposed earlier and the function is further developed here.

Occurrences of crossroads encountered along a route and their respective types were logged and taken into account - together with the traffic sign data gathered in a similar manner - in detecting change in the road environment. Statistical inference methodology relying on the $M D L$ principle was applied to the concrete change detection problem. $P H C D$ 's tuned to empirical data were set to work to detect the change. The process and the quality of change detection were demonstrated via examples.

In order to make the surrogate RoED ADAS method and subsystem usable in real automotive applications, an extensive collection of relevant data would be essential. It is imperative that the data comes from different regions and countries. Furthermore, as the built environment changes in time, the data collection trips need to be repeated.

The quality of the collected urban environment type data would improve by allowing one or more further urban environment type (e.g., for various agricultural areas, parks/ cemeteries). Furthermore, the data entry assistant should be allowed to log two different environment types for a route-segment (i.e., one for the left side and one for the right). 
Acknowledgments. The work presented was supported by the National Research, Development and Innovation Fund through project 'SEPPAC: Safety and Economic Platform for Partially Automated Commercial Vehicles' (VKSZ 14-1-2015-0125).

\section{References}

1. Piao, J., McDonald, M.: Advanced Driver Assistance Systems from Autonomous to Cooperative Approach. Transport Reviews, 28, 659-684 (2008)

2. Amditis, A., Pagle, K., Joshi, S., Bekiaris, E.: Driver-Vehicle-Environment Monitoring for On-board Driver Support Systems: Lessons Learned from Design and Implementation. Applied Ergonomics, 41, 225--235 (2010)

3. Fernandes, L.C., Souza, J.R., Pessin, G., Shinzato, P.Y., Sales, D., Mendes, C., Prado, M., Klaser, R., Magalhães, A.C., Hata, A., Pigatto, D., Branco, K.C., Grassi Jr. V., Osorio, F.S., Wolf, D.F.: CaRINA intelligent robotic car: architectural design and applications. Journal of Systems Architecture 60, 372--392 (2014)

4. Fazekas, Z., Gáspár, P.: Lane Information Traffic Signs - Important Sources of Information Mostly Ignored by Advanced Driver Assistance Systems. In: Proceedings of the International Scientific Conference on Modern Safety Technologies in Transportation. Kosice, Slovakia, 45-50 (2015)

5. OpenStreetMap contributors: Road Network in Hungary. URL: http://planet.openstreetmap. org, (2015)

6. Jiménez, F., Naranjo, J.E., Anaya, J.J., García, F. Ponz, A., Armingol, J.M.: Advanced Driver Assistance System for Road Environments to Improve Safety and Efficiency. Transportation Research Procedia 14, 2245--2254 (2016)

7. Fazekas, Z., Balázs, G., Gerencsér, L, Gáspár, P.: Inferring Actual Urban Road Environment from Traffic Sign Data using a Minimum Description Length Approach. Accepted for 20th EURO Working Group on Transportation Meeting, Budapest, Hungary (2017)

8. Fazekas, Z., Gerencsér, L, Gáspár, P.: Detecting Change in Road Environment via Analysis of Marked Point Processes Associated with Traffic Signs. Transportation Research Procedia, 22, 75--84 (2017)

9. Fazekas, Z., Balázs, G., Gerencsér, L, Gáspár, P.: Locating Roadwork Sites via Detecting Change in Lateral Positions of Traffic Signs Measured Relative to the Ego-car. Accepted for 20th EURO Working Group on Transportation Meeting, Budapest, Hungary (2017)

10. Page, E.S.: Continuous Inspection Schemes. Biometrika 41, 100--115 (1954)

11 Hinkley, D.: Inference about the Change-Point from Cumulative Sum Tests. Biometrika 58, 509--523 (1971)

12 Lorden, G.: Procedures for Reacting to a Change in Distribution. The Annals of Mathematical Statistics 42, 1897--1908 (1971)

13. Lai, T.L.: Sequential Changepoint Detection in Quality Control and Dynamical Systems. Journal of the Royal Statistical Society 57, 613--658 (1995)

14. Rissanen, J.: Modeling by Shortest Data Description. Automatica 14, 465--658 (1978)

15. Rissanen, J.: Stochastic Complexity in Statistical Inquiry. World Scientific Series in Computer Science, 15 (1998)

16. Baikovicius, J., Gerencsér, L.: Change Point Detection in a Stochastic Complexity Framework. In: Proceedings of 29th IEEE Conference on Decision and Control, 3554--3555 (1990)

17. Baikovicius, J., Gerencsér, L.: Change-point Detection as Model Selection. Informatica, 3, 3-20 (1992).

18 Fua, P., Hanson, A.J.: An Optimization Framework for Feature Extraction. Machine Vision and Applications, 4, 59--87 (1991) 